University of Wollongong

Research Online

Faculty of Engineering - Papers (Archive)

Faculty of Engineering and Information

Sciences

2003

\title{
LET dependence of the charge collection efficiency of silicon microdosimeters
}

I. Cornelius

University of Wollongong, iwan@uow.edu.au

Anatoly B. Rosenfeld

University of Wollongong, anatoly@uow.edu.au

R. Siegele

ANSTO, Australia

D. D. Cohen

ANSTO, Australia

Follow this and additional works at: https://ro.uow.edu.au/engpapers

Part of the Engineering Commons

https://ro.uow.edu.au/engpapers/26

\section{Recommended Citation}

Cornelius, I.; Rosenfeld, Anatoly B.; Siegele, R.; and Cohen, D. D.: LET dependence of the charge collection efficiency of silicon microdosimeters 2003.

https://ro.uow.edu.au/engpapers/26

Research Online is the open access institutional repository for the University of Wollongong. For further information contact the UOW Library: research-pubs@uow.edu.au 


\title{
LET Dependence of the Charge Collection Efficiency of Silicon Microdosimeters
}

\author{
Iwan M. Cornelius, Student Member, IEEE, Anatoly B. Rosenfeld, Senior Member, IEEE, Rainer Siegele, and \\ David D. Cohen
}

\begin{abstract}
A heavy ion microprobe was used to conduct ion beam induced charge (IBIC) collection imaging of silicon microdosimeters. The GEANT4 Monte Carlo toolkit was used to simulate these measurements to calculate ion energy loss in the device overlayer and energy deposition in the device sensitive volume. A comparison between experimental and theoretical results facilitated the calculation of charge collection efficiency profiles for several ions.
\end{abstract}

Index Terms-Charge collection efficiency, microbeam, microdosimetry, Monte Carlo.

\section{INTRODUCTION}

$\mathbf{S}$ ILICON microdosimeters, based on silicon-on-insulator (SOI) developments in the space radiation effects community, are being applied to hadron therapy applications [1]. The silicon microdosimeter is comprised of an array of microscopic, planar, pn junctions which are connected in parallel. By measuring the amount of charge generated by the traversal of an ion through the silicon sensitive volume one may calculate the energy lost to ionization by the ion. The ratio of this energy deposition event and the thickness of the sensitive volume gives an estimate of the ion LET.

As the electron-hole pairs generated in the sensitive volume are subject to recombination, it is necessary to quantify the fraction of charge that is collected, the charge collection efficiency (CCE). The CCE may then be used as a correction factor for measurements of ion LET. Previous research using a proton microprobe and semiconductor device simulation [2] indicated the charge collection process was dominated by the drift of charge carriers for ion strikes at the pn junction and by diffusion and lateral funneling for ion strikes outside the junction. The dominance of the diffusion process leads to increased recombination of electron hole pairs and results in a CCE which varies with ion strike location on the device (with an average value of 0.82 ). To circumvent this problem it was suggested to implement lateral isolation techniques to provide a fully depleted microscopic sensitive volume (as per reference [3]). Studies have shown that charge collection mechanisms, for instance the amount of recombination in the dense plasma of the ion track, are dependent

Manuscript received July 20, 2003; revisedNovember 3, 2003. This work was supported by the Australian Institute of Nuclear Science and Engineering.

I. M. Cornelius and A. B. Rosenfeld are with the Centre for Medical Radiation Physics, University of Wollongong, Wollongong, NSW 2522, Australia (e-mail: ic02@uow.edu.au).

R. Siegele and D. D. Cohen are with the Environment Division, Australian Nuclear Science and Technology Organization, Lucas Heights, NSW 2234, Australia.

Digital Object Identifier 10.1109/TNS.2003.820740

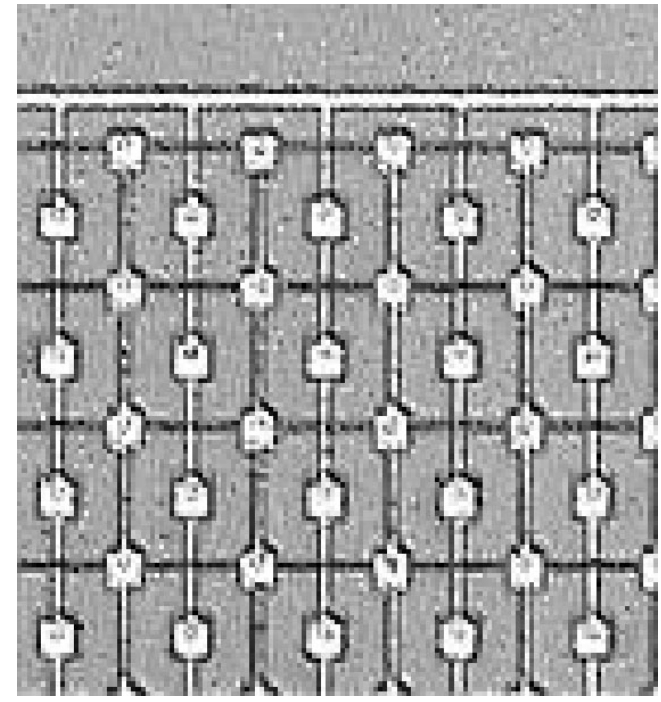

Fig. 1. Optical image of microdosimeter array showing aluminum contacts and tracks.

upon the initial distribution of electron hole pairs. This in turn is related to the ion LET or, more precisely, the ion track structure [4], [5]. The aim of this paper is to study the spatial variation of CCE for a single diode of the array, how the CCE varies with ion LET for LETs covering the range relevant to hadron therapy, and also the change in CCE with device bias and SOI thickness.

To determine the CCE a heavy ion microprobe was first used to conduct ion beam induced charge (IBIC) [6] collection imaging of the silicon microdosimeters. The GEANT4 Monte Carlo toolkit was then used to simulate these measurements to calculate energy deposition in the device sensitive volume taking into account ion energy loss in the device overlayer. By combining these results, profiles of CCE for a single diode of the array were formed.

\section{MethodS}

\section{A. Devices}

The silicon microdosimeters being studied are manufactured on bonded p-type silicon on insulator (SOI) wafers of thicknesses 2,5 , and $10 \mu \mathrm{m}$ with a Boron impurity concentration of $1.5 \times 10^{15} \mathrm{~cm}^{-3} \cdot n^{+}$and $p^{+}$silicon regions are constructed with Arsenic and Boron implantations at $30 \mathrm{keV}$ at a fluence of $5 \times 10^{15} \mathrm{~cm}^{-2}$. Each pn junction has an area of $8 \times 8 \mu \mathrm{m}^{2}$ and the total size of each diode cell is $30 \times 30 \mu \mathrm{m}^{2}$. The array is comprised of $120 \times 40$ diodes connected in parallel (see Fig. 1). 


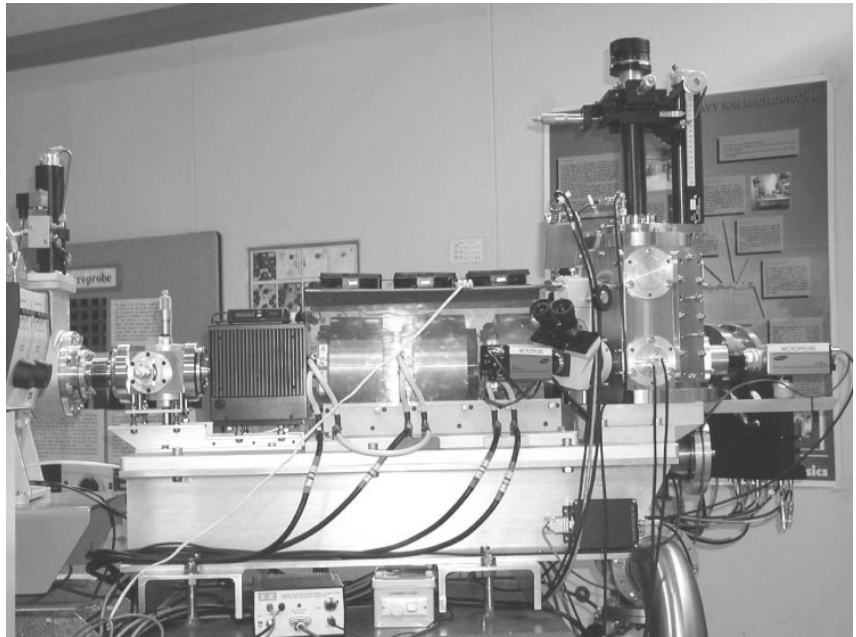

Fig. 2. ANSTO heavy ion microprobe end station.

\section{B. IBIC Experiments}

Experiments were carried out on the heavy ion microprobe at the Australian Nuclear Science and Technology Organization (ANSTO) [7] (see Fig. 2). The microprobe was used to produce focussed beams scanned over an area of approximately $100 \times 100 \mu \mathrm{m}^{2}$. From Scanning Transmission Ion Microscopy of a copper test grid [8], the beam diameter was found to be $1.5 \mu \mathrm{m}$. The ion atomic numbers and energies used in the IBIC experiments were; $3 \mathrm{MeV}$ protons, $9 \mathrm{MeV}$ alphas, and $25 \mathrm{MeV}$ carbon ions. These correspond to LETs and ranges in silicon of $19.7 \mathrm{keV} / \mu \mathrm{m}$ and $92.1 \mu \mathrm{m}, 97.6 \mathrm{keV} / \mu \mathrm{m}$ and $59.1 \mu \mathrm{m}$, and $820 \mathrm{keV} / \mu \mathrm{m}$ and $25.7 \mu \mathrm{m}$, respectively. The ion beam induced charge collection setup [9] consists of a charge sensitive preamplifier (Amptek A250) mounted on a PC250 test board. A 10 pin electrical feed-through conveys the preamplifier power supply, device bias, test pulse signal, and charge collection signal to and from the target chamber. The preamplifier output signal is amplified and shaped by a spectroscopy amplifier and passed to an ADC channel of the data acquisition system in coincidence with signals indicating the beam position. Calibration of the IBIC system is achieved by measuring the variation of pulse height from a fully depleted, ion implanted detector with angle of incidence of a $3 \mathrm{MeV}$ proton beam [10]. In this manner the charge collection pulse height is calibrated in terms of energy lost in silicon.

IBIC measurements were carried out on microdosimeters with 5 and $10 \mu \mathrm{m}$ thick SOI layers at a reverse bias of $0,5,10$, and $20 \mathrm{~V}$. For each IBIC measurement a frequency distribution of energy deposition events is produced along with a $256 \times$ 256 pixel image of the median energy event. The median is used in IBIC to reduce sensitivity to outlying events such as those resulting from scattered ions [8]. It is well known that displacement damage induced by IBIC imaging can lead to degradation of minority carrier lifetime over the course of measurement [11]. To minimize this effect, the ion fluence used for each analysis was limited to the point at which a noticeable change in the frequency distribution occurred. This varied from $2-16 \mu \mathrm{m}^{-2}$ for carbon ions and protons, respectively. This action results in degraded image statistics so in order to improve

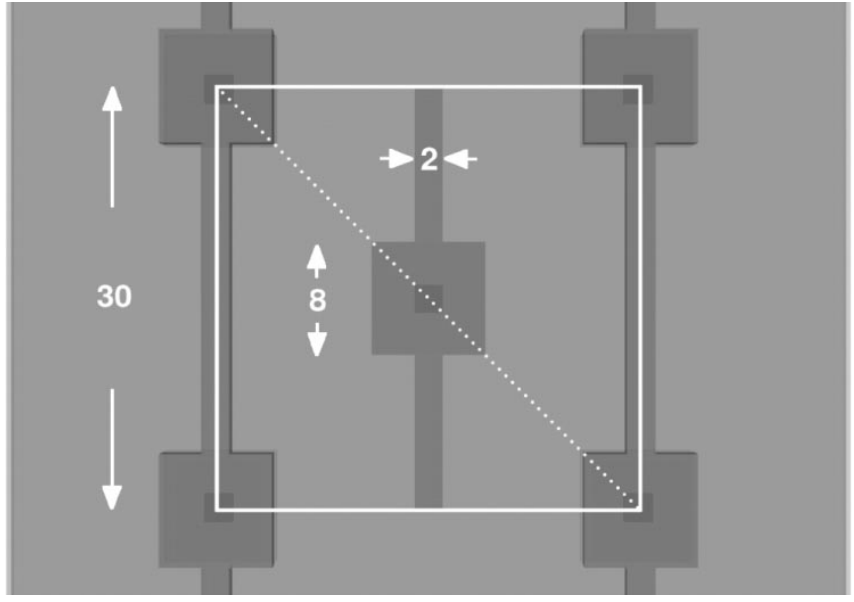

Fig. 3. Geometry used for GEANT4 simulations. Solid square indicates simulated IBIC scan area. Dotted line indicates orientation of CCE profiles.

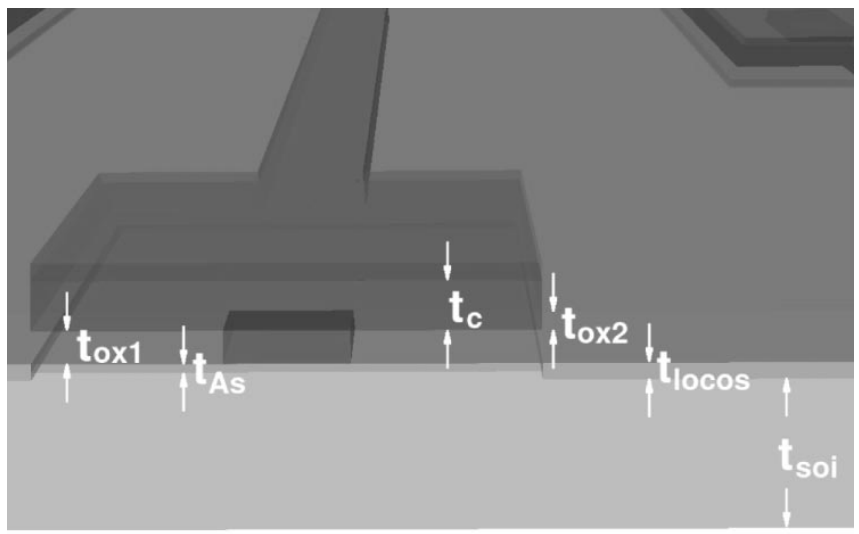

Fig. 4. Cross section of device geometry used for GEANT4 simulations showing thicknesses of device regions.

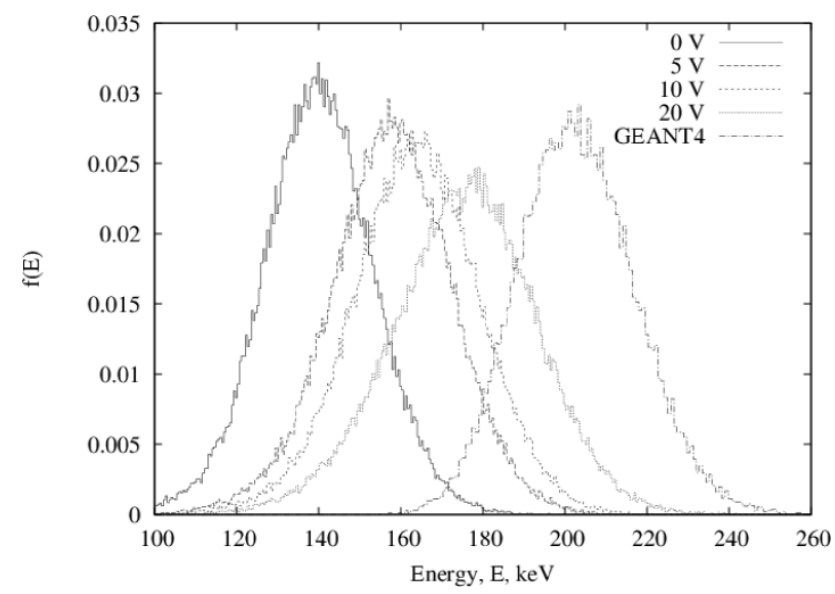

Fig. 5. Simulated and experimental IBIC spectra for $3 \mathrm{MeV}$ proton scan of $10 \mu \mathrm{m}$ device.

statistics it was necessary to reduce the number of pixels in the image to $64 \times 64$ by calculating the median over an $8 \times 8$ pixel matrix. The error at each pixel is calculated as the standard deviation of values from the median value. We wish to derive a two dimensional image of the median charge collected for a single diode. To achieved this the entire image is translated to place a single $30 \times 30 \mu \mathrm{m}^{2}$ diode in the corner of the image 


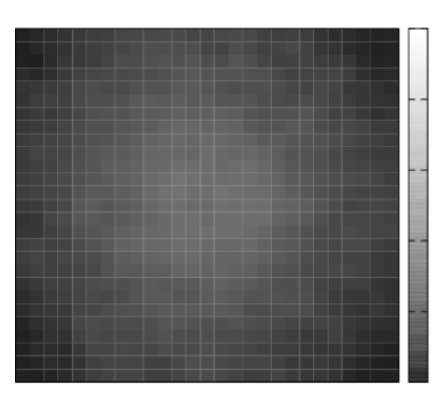

220
200
180
160
140
120

120
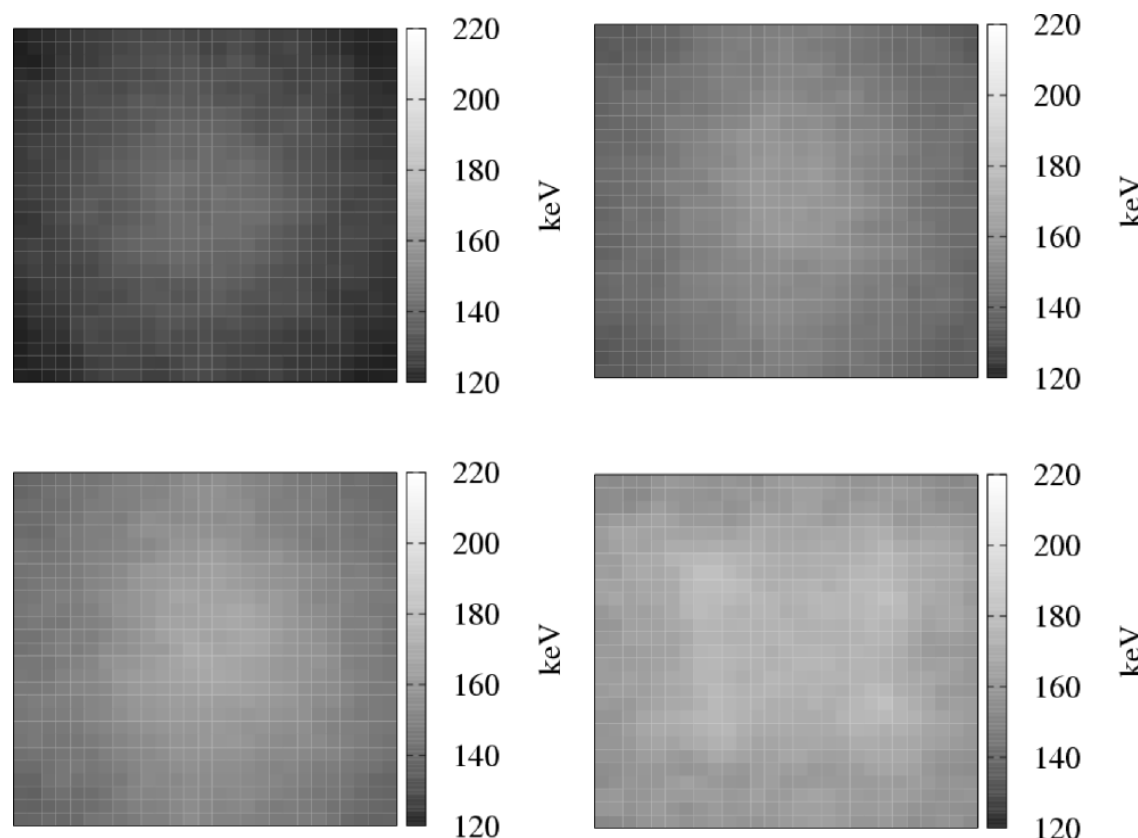

220
200
180
160
140
120

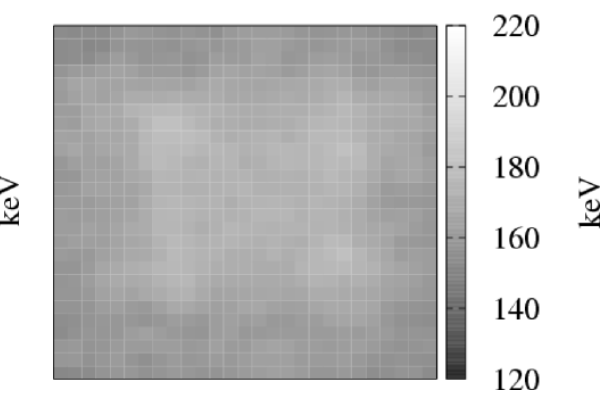

Fig. 6. Experimental IBIC median energy images for $3 \mathrm{MeV}$ proton scan of $10 \mu \mathrm{m}$ device at bias of $0 \mathrm{~V}$ (top left), $5 \mathrm{~V}$ (top right), $10 \mathrm{~V}$ (bottom left), and $20 \mathrm{~V}$ (bottom right).

then cropped to produce an $X \times X$ pixel image of the median energy for a single diode, $E_{\exp }(x, y) . X$ varies from 23-28 depending upon the ion atomic numbers and energy used.

\section{GEANT4 Simulations}

To facilitate CCE calculations these IBIC measurements were simulated with the GEANT4 Monte Carlo toolkit [12]. Images of energy deposition for a single diode of the silicon microdosimeter were calculated with the ions and device thicknesses used in the experiments.

A single cell of the diode array was modeled by combining a system of right angle parallelapiped (RPP) volumes using the Boolean (union, intersection or subtraction) operations available with the GEANT4 toolkit. The geometry is illustrated in Figs. 3 and 4 and is based on fabrication information and secondary ion mass spectrometry data from Fujitsu Research Laboratories [13]. The SOI wafer is modeled as a cuboid with dimensions $60 \times 60 \times t_{\text {soi }} \mu \mathrm{m}^{3}$, where $t_{\text {soi }}=5$ or $10 \mu \mathrm{m}$ (see Fig. 4). A central $n^{+}$region of dimensions $8 \times 8 \times t_{A s} \mu \mathrm{m}^{3}$ is considered where $t_{A s}=0.1 \mu \mathrm{m}$ is the implantation depth of As ions. $p^{+}$regions of dimensions $8 \times 8 \times t_{B} \mu \mathrm{m}^{3}$, where $t_{B}=0.25 \mu \mathrm{m}$ is the Boron implantation depth are considered with centers at each corner of the cell (see Fig. 3). The LOCOS (local consumption of silicon), silicon oxide layer is modeled as an RPP of thickness $t_{\text {locos }}=0.200 \mu \mathrm{m}$. A silicon oxide overlayer of thickness $t_{o x 1}=0.400 \mu \mathrm{m}$ is considered above the SOI wafer. Aluminum contacts penetrating this layer, of dimensions $2 \times 2 \times t_{o x 1} \mu \mathrm{m}^{3}$ are placed above each $n^{+}$and $p^{+}$ region. Aluminum volumes over these contacts of dimensions $8 \times 8 \times 1 \mu \mathrm{m}^{3}$ are considered and tracks connecting these volumes are described by RPPs of dimensions $11 \times 2 \times 1 \mu \mathrm{m}^{3}$. Finally, a thickness $t_{o x 2}=0.215 \mu \mathrm{m}$ of silicon oxide is considered over all volumes.

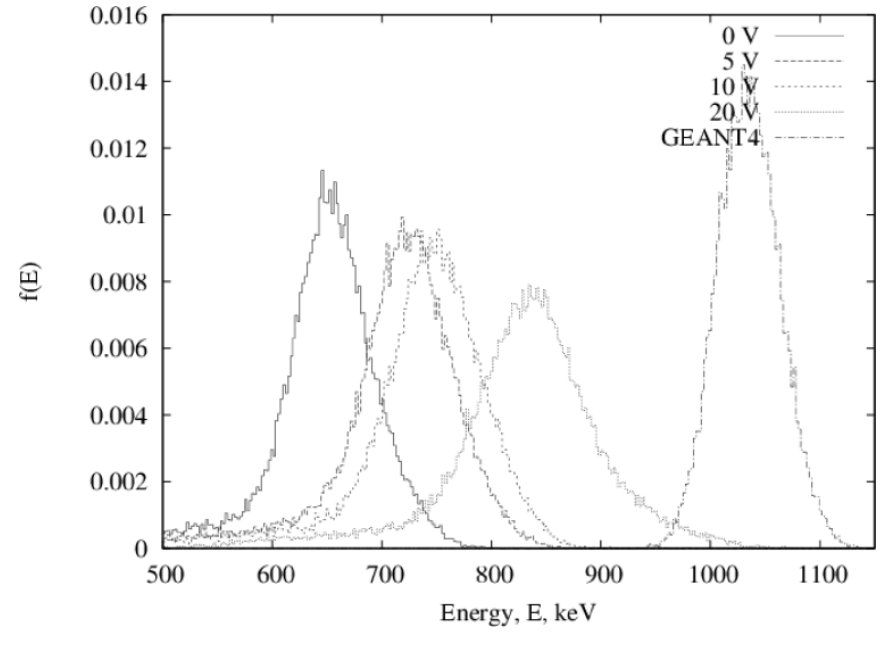

Fig. 7. Simulated and experimental IBIC spectra for $9 \mathrm{MeV}$ helium scan of $10 \mu \mathrm{m}$ device.

The ion beam is defined as normally incident on the device and raster scanned across a $30 \times 30 \mu \mathrm{m}^{2}$ area of the diode cell centered on the $n^{+}$region (see white square in Fig. 3). The number of pixels in each scan is identical to the number in the corresponding experimental charge collection image (i.e., $X \times X$ in Section II-B). At each pixel, $x$ and $y$ coordinates of 50 ions are randomly sampled from a $1.5 \mu \mathrm{m}^{2}$ area to model the beam spot size. Ions are transported through the geometry taking into account ionization of atomic electrons and scatter interactions with nuclei using existing GEANT4 physics models [12]. For each ion history, energy lost to ionization in the SOI layer is calculated and output to file along with the beam position. This output file is analyzed and the median energy event for each pixel is calculated as $E \sim(x, y)$. The error is determined as the standard deviation of energy events from the median value. 

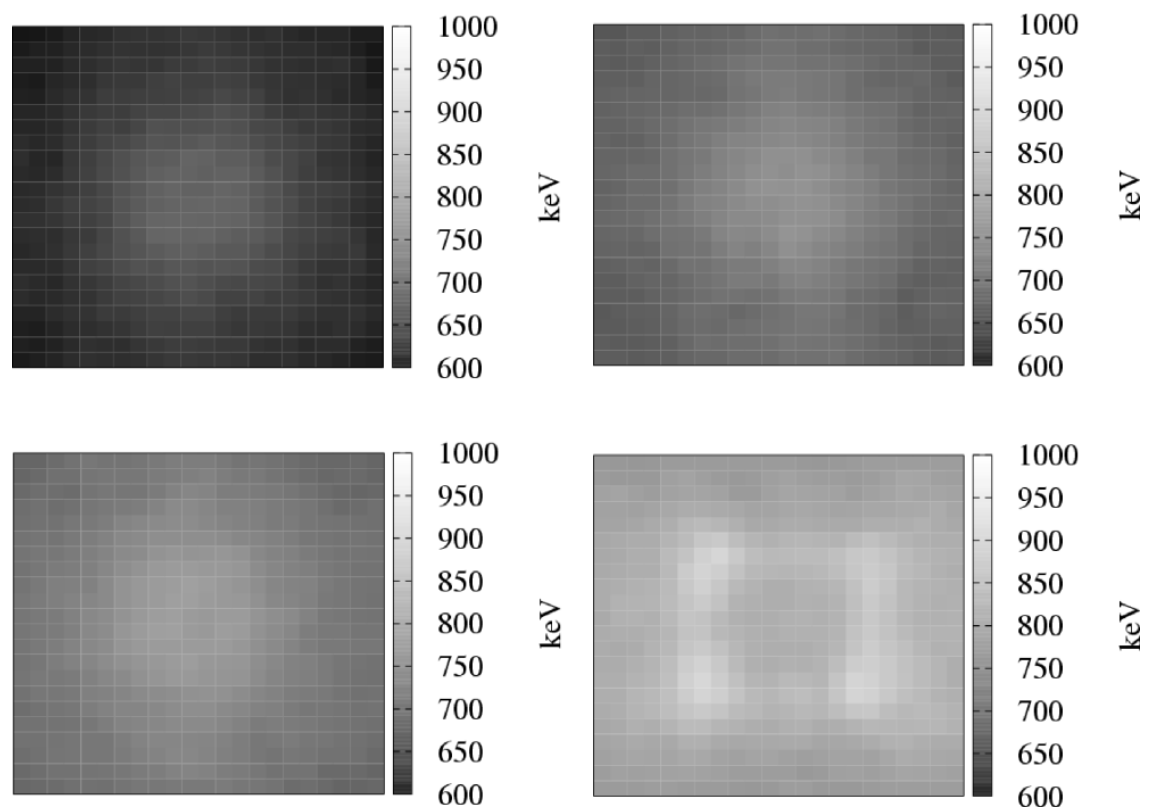

Fig. 8. Experimental IBIC median energy image for $9 \mathrm{MeV}$ helium scan of $10 \mu \mathrm{m}$ device at bias of $0 \mathrm{~V}$ (top left), $5 \mathrm{~V}$ (top right), $10 \mathrm{~V}$ (bottom left), and $20 \mathrm{~V}$ (bottom right).

\section{Charge Collection Efficiency Profiles}

A charge collection efficiency map is formed from the simulated and experimental median energy images as

$$
\operatorname{CCE}(x, y)=\frac{E_{\exp }(x, y)}{E_{\sim}(x, y)} .
$$

The relative error of the $\mathrm{CCE}$ at each pixel is calculated as

$$
\frac{\delta \mathrm{CCE}(x, y)}{\operatorname{CCE}(x, y)}=\sqrt{\left(\frac{\delta E_{\sim}(x, y)}{E_{\sim}(x, y)}\right)^{2}+\left(\frac{\delta E_{\exp }(x, y)}{E_{\exp }(x, y)}\right)^{2}} .
$$

To draw a comparison of CCE for the ions used a profile was calculated along a diagonal of the diode connecting $p^{+}$regions (indicated by dotted line in Fig. 3).

\section{RESULTS AND DISCUSSION}

\section{A. IBIC Experiments}

Fig. 5 shows the experimental energy spectra obtained from the $3 \mathrm{MeV}$ proton scan of the $10 \mu \mathrm{m}$ SOI device. Each spectrum is composed of a single peak which shifts to higher energies with an increase in applied bias. Fig. 6 shows the median energy maps derived from the IBIC measurements. For applied bias of 0,5 , and $10 \mathrm{~V}$ the image is characterized by a maximum at the centre of the diode with a subtle decrease toward the diode periphery. This reflects a low sensitivity to device overlayer for protons and a diffusion length much greater than diode dimensions. This maximum value increases with applied bias reflecting an increase in depletion layer thickness, consequent decrease in charge collection time and hence electron hole recombination. The image characteristics change for a bias of $20 \mathrm{~V}$, in which case the maximum median energy event occurs at the corners of the $n^{+}$region. The probable mechanism for this enhanced charge collection is the occurrence of high electric fields in these regions at $20 \mathrm{~V}$ bias.

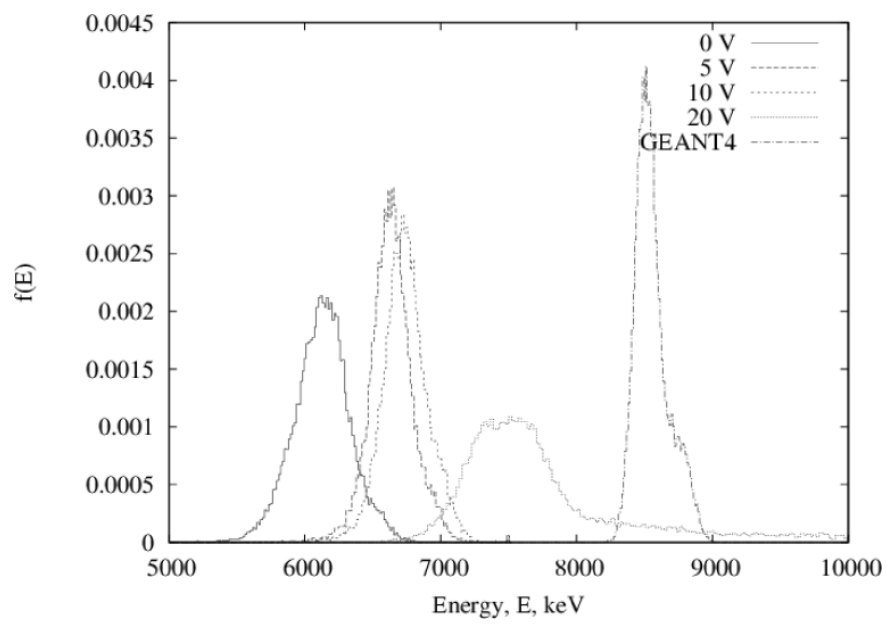

Fig. 9. Simulated and experimental IBIC spectra for $25 \mathrm{MeV}$ carbon scan of $10 \mu \mathrm{m}$ device at given bias.

Fig. 7 shows the experimental energy spectra obtained from the $9 \mathrm{MeV}$ helium scan of the $10 \mu \mathrm{m}$ SOI device. The spectra occur at higher energies owing to the increased LET for $9 \mathrm{MeV}$ helium and display a similar dependence on applied bias as for the proton scan. Fig. 8 shows the median energy images are very similar to the case for proton results, including the amplification effect at $20 \mathrm{~V}$.

Fig. 9 shows the experimental energy spectra obtained from the $25 \mathrm{MeV}$ carbon scan of the $10 \mu \mathrm{m}$ SOI device. Again spectra occur at higher energies owing to the increased LET for $25 \mathrm{MeV}$ carbon and display a similar dependence on applied bias as for the proton and helium scans. Fig. 10 shows the median energy images which illustrate an increased sensitivity to device overlayer. Clearly visible in the 0,5 , and $10 \mathrm{~V}$ images are areas of increased energy deposition corresponding to the aluminum contact overlying the $n^{+}$region and aluminum tracks connecting these contacts. The amplification effect at $20 \mathrm{~V}$, both in spectrum 

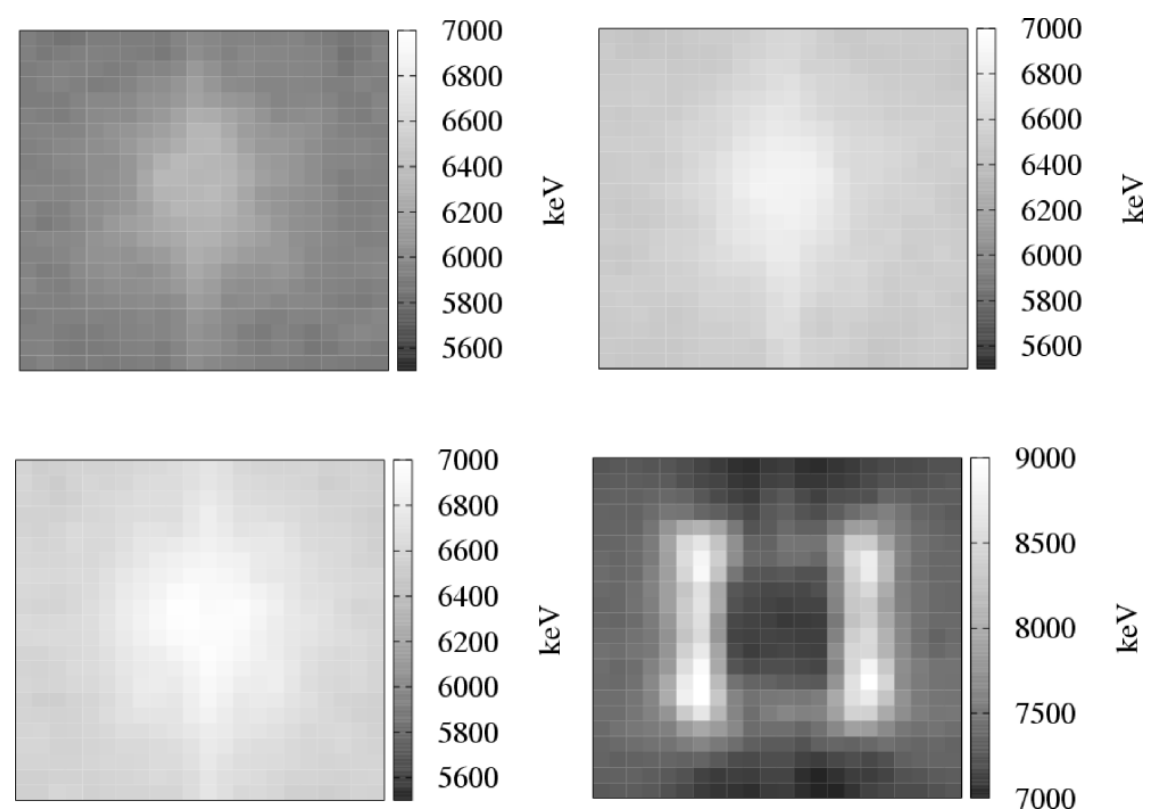

7000
6800
6600
6400
6200
6000
5800
5600

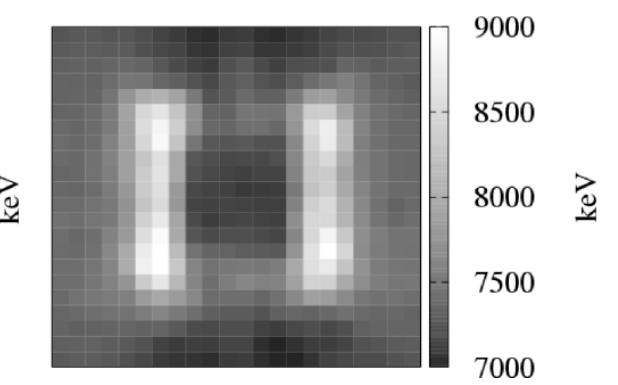

Fig. 10. Experimental IBIC median energy image for $25 \mathrm{MeV}$ carbon scan of $10 \mu \mathrm{m}$ device at bias of $0 \mathrm{~V}$ (top left), $5 \mathrm{~V}$ (top right), $10 \mathrm{~V}$ (bottom left), and $20 \mathrm{~V}$ (bottom right).
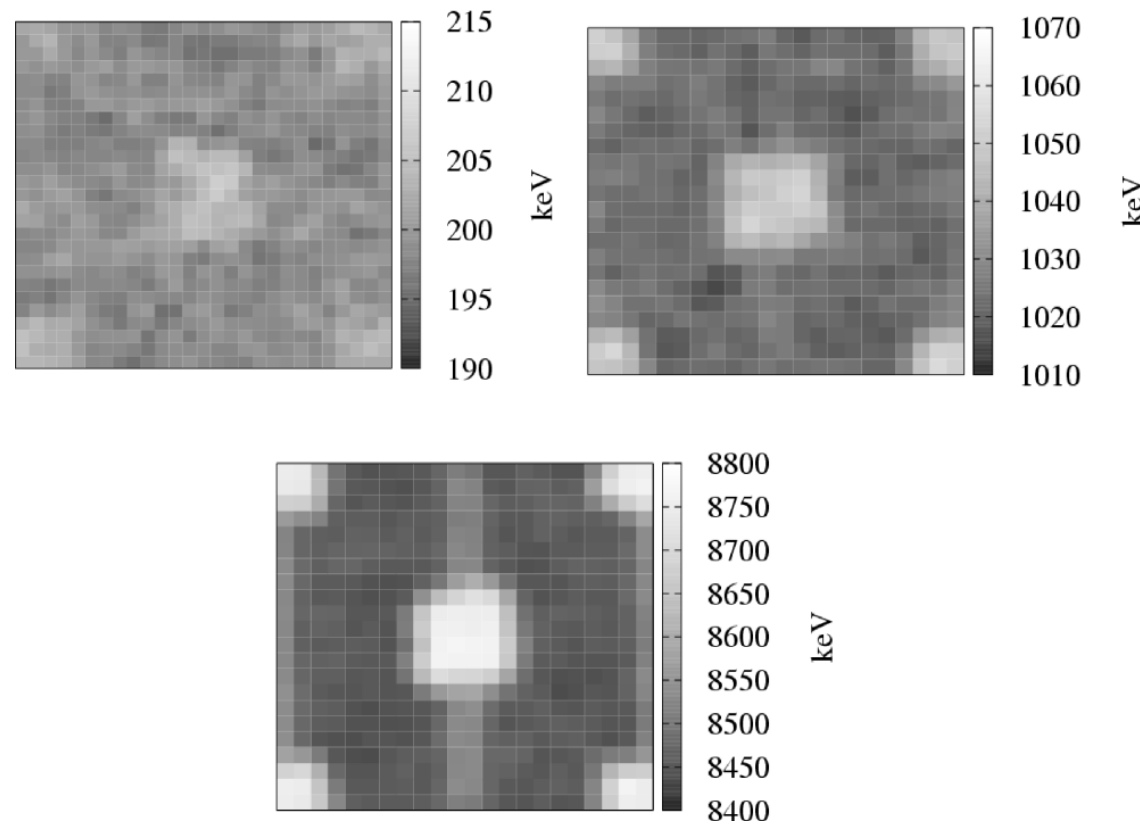

Fig. 11. Simulated IBIC image for $10 \mu \mathrm{m}$ device with $3 \mathrm{MeV}$ protons (top left), $9 \mathrm{MeV}$ helium (top right), and $25 \mathrm{MeV}$ carbon (bottom).

and median energy image is more pronounced than for $3 \mathrm{MeV}$ protons and $9 \mathrm{MeV}$ helium.

\section{B. GEANT4 Simulations}

Fig. 11 shows the median energy deposition event at each pixel of the simulated proton, helium, and carbon IBIC scans of the $10 \mu \mathrm{m}$ device. As expected there is an increase in energy deposition for ion strikes over the $p^{+}$and $n^{+}$regions owing to energy loss in the aluminum contacts and highly doped silicon regions and a thicker SOI layer to the rest of the sensitive volume owing to the LOCOS regions. The image contrast increases with increasing LET owing to a greater sensitivity to energy loss in the overlayer structures. For $25 \mathrm{MeV}$ carbon the aluminum tracks connecting contacts are clearly visible (as was seen in experimental results of Fig. 10).

\section{Charge Collection Efficiency Profiles}

Figs. 12-14 show CCE profiles for the $10 \mu \mathrm{m}$ device at bias of 0,10 , and $20 \mathrm{~V}$. At $0 \mathrm{~V}$ the charge collection efficiency profile lies within the range $0.5-0.8$, increasing to $0.6-0.9$ for $10 \mathrm{~V}$ and $0.7-1.1$ for $20 \mathrm{~V}$. The CCE of greater than unity for carbon ions at $20 \mathrm{~V}$ suggest a high field avalanche multiplication effect. A similar effect in thin epitaxial pn junctions was observed by Knudson et al. [14]. The errors of the CCE increases with a decreasing $\mathrm{Z}$ of the ion beam used. This is due to the higher cross section for nuclear scatter and hence higher variation in 


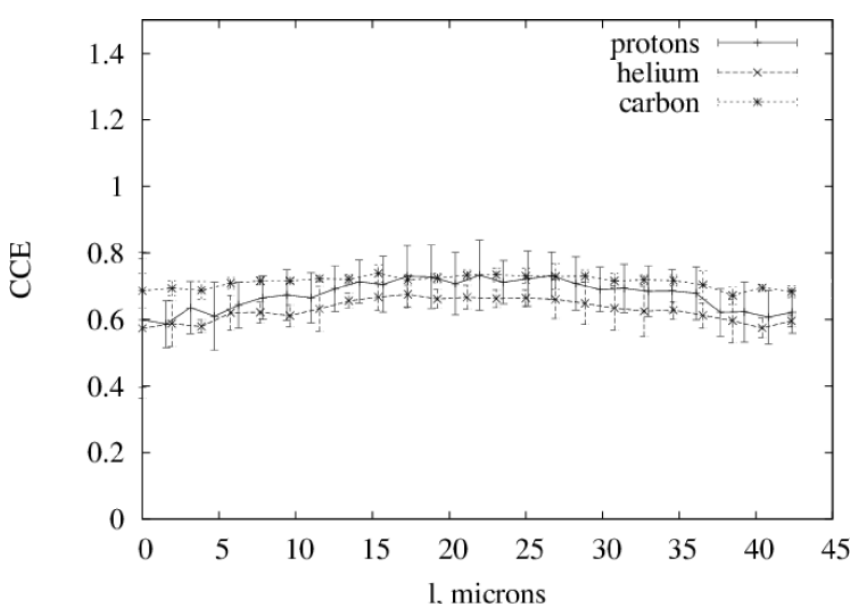

Fig. 12. Charge collection efficiency profiles for $10 \mu \mathrm{m}$ device at bias of $0 \mathrm{~V}$. 1 is distance along diagonal of Fig. 3.

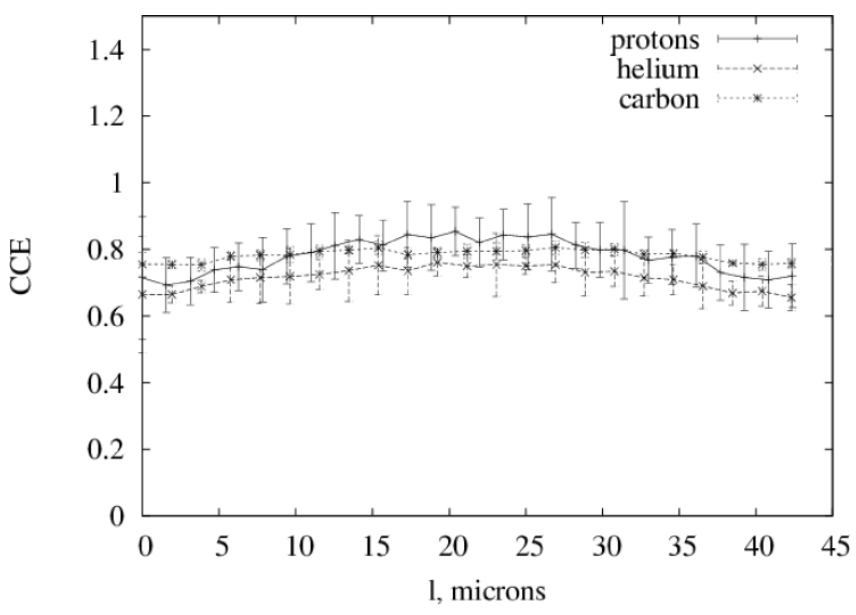

Fig. 13. Charge collection efficiency profiles for $10 \mu \mathrm{m}$ device at bias of $10 \mathrm{~V}$. 1 is distance along diagonal of Fig. 3.

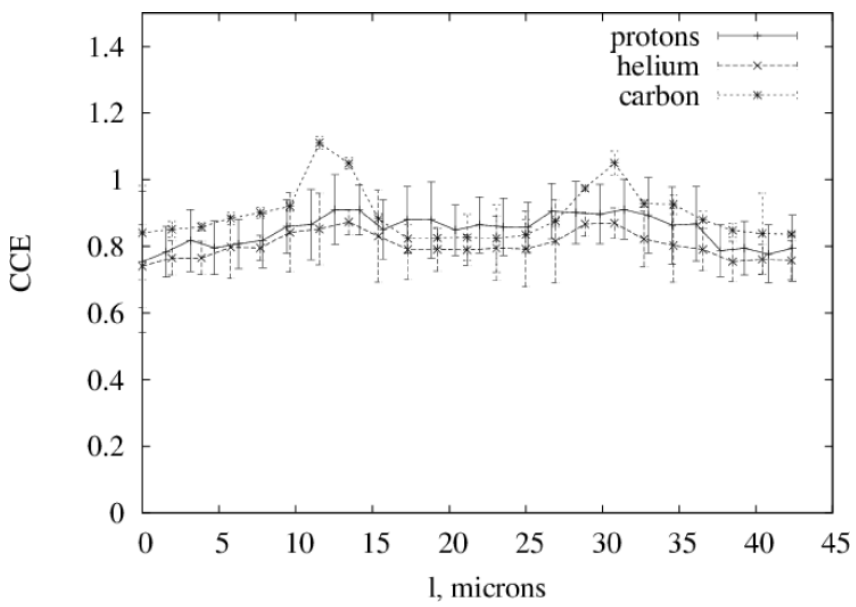

Fig. 14. Charge collection efficiency profiles for $10 \mu \mathrm{m}$ device at bias of $20 \mathrm{~V}$. 1 is distance along diagonal of Fig. 3.

path length through the sensitive volume. The CCE values of carbon are seen to exceed those of helium, although the LET is much greater. This may be due to enhanced charge funnelling from below the depletion region.

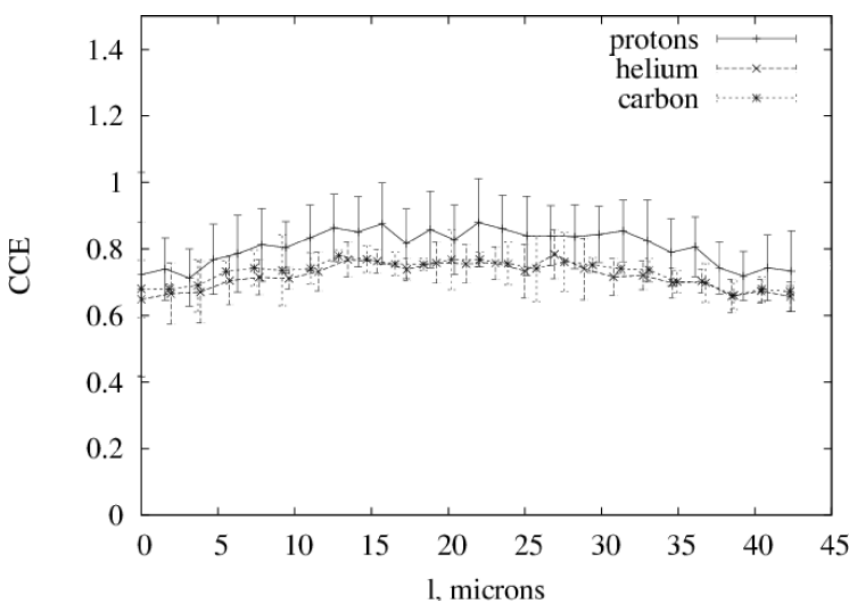

Fig. 15. Charge collection efficiency profiles for $5 \mu \mathrm{m}$ device at bias of $0 \mathrm{~V} .1$ is distance along diagonal of Fig. 3.

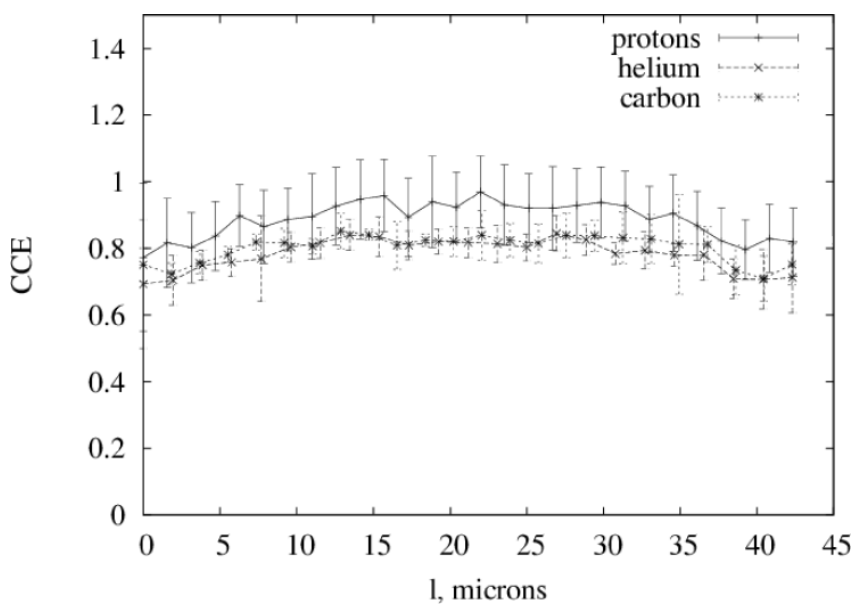

Fig. 16. Charge collection efficiency profiles for $5 \mu \mathrm{m}$ device at bias of $10 \mathrm{~V}$. 1 is distance along diagonal of Fig. 3.

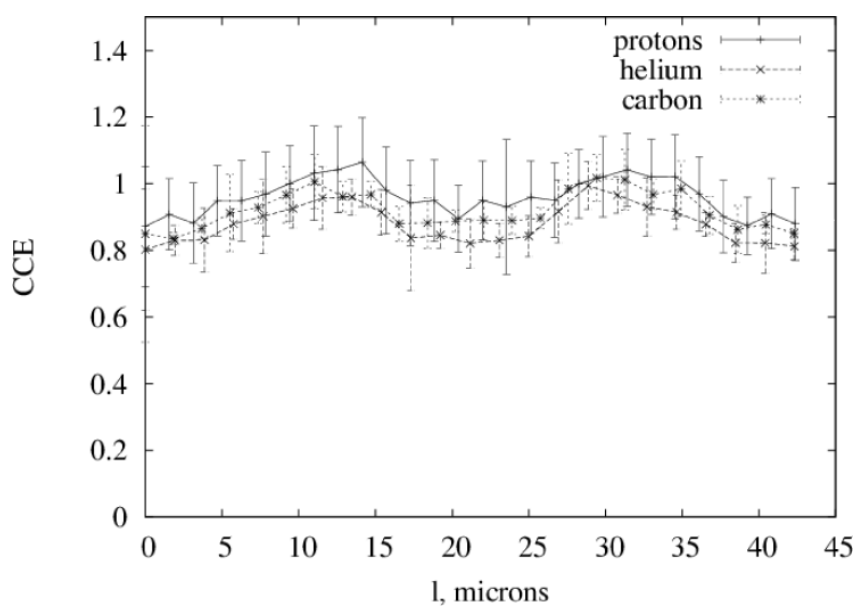

Fig. 17. Charge collection efficiency profiles for $5 \mu \mathrm{m}$ device at bias of $20 \mathrm{~V}$. 1 is distance along diagonal of Fig. 3 .

Figs. 15-17 show CCE profiles for the $5 \mu \mathrm{m}$ device at bias of 0,10 , and $20 \mathrm{~V}$. CCE values exceed those of the $10 \mu \mathrm{m}$ device at the same bias, a result of the increased fraction of SOI layer depleted at the same voltage. The charge amplification effect is greater than observed for the $10 \mu \mathrm{m}$ device and values 
exceeding unity extend approximately $5 \mu \mathrm{m}$ from the edge of the $n^{+}$region.

\section{CONCLUSION}

The CCE of a silicon microdosimeter has been calculated for a number of ions. This was achieved via comparison of experimental IBIC measurements and simulations with the Geant4 Monte Carlo toolkit. Values of CCE for the ions studied were seen to agree within statistical uncertainties with a general increase with decreasing device thickness and increase with increasing device bias. An amplification effect was observed for device bias of $20 \mathrm{~V}$, a possible mechanism for this effect is high field avalanche multiplication at corners of the depletion region. Recent studies into charge collection in SOI structures have shown that significant charge collection can be induced by displacement currents across the oxide [15]. Simulation of the charge collection processes for these devices are planned to gain insight into the physical origins of the multiplication effect and to study the importance of induced displacement currents.

A potential source of systematic error in this method of CCE calculation is from uncertainty in device composition and geometry and the approximation with a system of RPP volumes. A more accurate method of geometry definition is possible with GEANT4 by importing solid definitions from STEP compliant CAD systems.

The implications of this study for actual silicon microdosimetry measurements are that although the measurement of ion LET must be corrected by the CCE of the device, it is not necessary to apply an LET dependent correction factor for hadron therapy applications. These results do emphasize the need to minimize device overlayers and implement lateral isolation in next generation devices. High electric fields should also be avoided as amplification effects complicate charge collection and are seen to vary in magnitude with ion LET.

\section{ACKNOWLEDGMENT}

The authors would like to acknowledge the efforts of the Accelarator Operations Team, Environment Division, ANSTO, and the support of the Australian Institute of Nuclear Science and Engineering. They would also like to thank the GEANT4 collaboration for the Monte Carlo toolkit and members of the online users forum for their support.

\section{REFERENCES}

[1] A. Rosenfeld, P. Bradley, I. Cornelius, G. Kaplan, B. Allen, J. Flanz, M. Goitein, A. V. Meerbeeck, J. Schubert, J. Bailey, Y. Tabkada, A. Maruhashi, and Y. Hayakawa, "New silicon detector for micro-dosimetry applications in proton therapy," IEEE Trans. Nucl. Sci., vol. 47, pp. 1386-1394, Aug. 2000.

[2] P. Bradley, A. Rosenfeld, K. Lee, D. Jamieson, and S. Satoh, "Charge collection and radiation hardness of a SOI microdosimeter for space and medical applications," IEEE Trans. Nucl. Sci., vol. 45, pp. 2700-2710, Dec. 1998.

[3] T. Colladant, V. Ferlet-Cavrois, O. Musseau, A. L'Hoir, A. Campbell, S. Buchner, A. Knudson, D. McMorrow, B. Fischer, M. Schogl, and D. Lewis, "Charge collection studies of SOI diodes," IEEE Trans. Nucl. Sci., vol. 49, pp. 1372-1376, June 2002.

[4] W. Stapor, P. McDonald, A. Knudson, and A. Campbell, "Charge collection in silicon for ions of different energy but same linear energy transfer (LET)," IEEE Trans. Nucl. Sci., vol. 35, pp. 1585-1590, Dec. 1988.

[5] P. Dodd, F. Sexton, and P. Winokur, "Three-dimensional simulation of charge collection and multiple-bit upset in Si devices," IEEE Trans. Nucl. Sci., vol. 41, pp. 2005-2017, Dec. 1994.

[6] F. Sexton, "Microbeam studies of single-event effects," IEEE Trans. Nucl. Sci., vol. 43, pp. 687-695, Apr. 1996.

[7] R. Siegele, D. Cohem, and N. Dytlewski, "The ANSTO high energy heavy ion microprobe," Nucl. Instrum. Methods Phys. Res. B, vol. 158, pp. 31-38, 1999.

[8] M. Breese, D. Jamieson, and P. King, Materials Analysis Using a Nuclear Microprobe. New York: Wiley, 1996.

[9] I. Cornelius, R. Siegele, A. Rosenfeld, and D. Cohen, "Ion beam induced charge collection imaging of a silicon microdosimeter using a heavy ion microprobe," Nucl. Instrum. Methods Phys. Res. B, vol. 184, pp. 135-157, 2001.

[10] C. Yang, D. Jamieson, S. Hearne, C. Parkes, B. Rout, E. Gauja, A. Dzurak, and R. Clark, "Ion-beam-induced-charge characterization of particle detectors," Nucl. Instrum. Methods Phys. Res. B, vol. 190, pp. 212-216, 2002.

[11] F. Sexton, K. Horn, B. Doyle, M. Shaneyfelt, and T. Meisenheimer, "Effects of ion damage on IBICC and SEU imaging," IEEE Trans. Nucl. Sci., vol. 42, pp. 1940-1946, Dec. 1995.

[12] ,GEANT4.http://wwwinfo.cern.ch/asd/geant4/geant4.html [Online]

[13] P. Bradley, "The Development of a Novel Silicon Microdosimeter for High LET Radiation Therapy ," Ph.D. dissertation, Univ. Wollongong, Wollongong, NSW, Australia, 2000.

[14] A. Knudson, A. Campbell, J. Hauser, M. Jessee, W. Stapor, and P. Shapiro, "Charge transport in test structures," IEEE Trans. Nucl. Sci., vol. NS-30, pp. 4486-4492, Dec. 1983.

[15] J. Schwank, P. Dodd, M. Shaneyfelt, G. Vizkelethy, B. Draper, T. Hill, D. Walsh, G. Hash, B. Doyle, and F. McDaniel, "Charge collection in SOI capacitors and circuits and its effect on SEU hardness," IEEE Trans. Nucl. Sci., vol. 49, pp. 2937-2947, Dec. 2002. 